\title{
Efficient field emission from Li-salt functionalized multiwall carbon nanotubes on flexible substrates
}

\author{
S. M. Lyth, ${ }^{\text {a) }}$ R. A. Hatton, and S. R. P. Silva \\ Nano-Electronics Centre, Advanced Technology Institute, University of Surrey, Guildford, Surrey GU2 7XH, \\ United Kingdom
}

(Received 18 October 2006; accepted 1 December 2006; published online 5 January 2007)

\begin{abstract}
The authors report extremely low electron field emission thresholds of $0.25 \mathrm{~V} / \mu \mathrm{m}$ from lithium salt functionalized multiwall carbon nanotubes adhered to carbon fiber fabric. Crucially, these nanostructured field emitters are flexible, air stable, and produced via a low cost dip-processing method using an aqueous nanotube ink, whereupon the nanotubes spontaneously assemble onto a surface oxidized carbon fiber matrix to form dense mats. The very low emission threshold is rationalized in terms of the morphology of the nanotube mats and the relatively low work function of lithium salt derivated carbon nanotubes. (C) 2007 American Institute of Physics.
\end{abstract}

[DOI: 10.1063/1.2430091]

Carbon nanotubes (CNTs) have excellent field emission (FE) properties $^{1-4}$ owing to their high aspect ratio and high electrical conductivity. ${ }^{5}$ Consequently, CNTs have been extensively investigated as cold cathodes for lighting and flat panel displays. ${ }^{6-8}$ The realization of low cost, large area CNT cathodes is crucial if devices are to become contenders in the marketplace and provided the large area driver circuitry can also be realized, a winning combination is at hand. ${ }^{9}$ In this letter we report a method for homogeneously applying multiwall carbon nanotubes (MWNTs) over large areas onto carbon fiber fabric via solution processing at room temperature. We report a substantial improvement in the field emission properties after converting carboxylic acid groups on the oxidized MWNT surface to the carboxylate salt of lithium via facile cation exchange. It is envisaged that this technique could be scaled up to a roll-to-roll industrial process for producing truly large area, cheap FE substrates with excellent, homogeneous emission properties.

The graphitic surface of MWNT renders them hydrophobic and it is notoriously difficult to obtain a stable dispersion in common organic solvents without chemical derivatization of the outer surface. A simple and common method to form stable aqueous dispersions is to reflux MWNT in a concentrated nitric and sulfuric acid mixture resulting in the formation of oxygen containing moieties including carboxylic acid groups $(-\mathrm{CO} \cdot \mathrm{OH})$ attached to the MWNT ends and sidewalls. ${ }^{0}$ Polar oxygen containing moieties such as carboxylic groups readily interact with water molecules via hydrogen bonding. Functionalization via acid treatment also serves to purify MWNTs removing catalytic particles and amorphous carbon. For the sake of clarity, acid oxidized MWNT will herein be referred to as o-MWNT.

The work function of materials plays a crucial role in their field emission properties. From the well-known FowlerNordheim equation, ${ }^{11}$ it is evident that reducing the work function should result in an exponential increase in current density, at a given electric field. Therefore, for cold cathode applications it is desirable to minimize the emitter work function without compromising the cathode stability towards oxidation. Several groups have attempted to reduce the work

${ }^{a)}$ Electronic mail: s.lyth@surrey.ac.uk function of CNTs for field emission by coating, intercalation with low work function metals and chemical functionalization, with mixed results. ${ }^{12-17}$ We have recently shown that the work function of acid oxidized MWNT can be dramatically reduced upon the exchange of protons associated with acidic surface groups with alkali metal cations. ${ }^{18}$ Crucially, this process does not change the MWNT dimensions and does not compromise the stability of the MWNT.

High purity (95\%) MWNTs with a nominal diameter of $10 \mathrm{~nm}$ were purchased from Nanocyl and ultrasonically dispersed in a 3:1 concentrated sulfuric and nitric acid mixture for $10 \mathrm{~min}$. The mixture was refluxed at $110{ }^{\circ} \mathrm{C}$ for $40 \mathrm{~min}$ before diluting with de-ionized water. Large particles and agglomerates were removed from the solution by centrifuging. The centrifuged liquid was carefully decanted, filtered through a $0.2 \mu \mathrm{m}$ polycarbonate membrane, and washed with de-ionized water until a slurry with $p \mathrm{H}$ 6-7 was obtained. This slurry was added to a small amount of deionized water, which was sonicated to disperse the tubes. The resulting product was a stable o-MWNT dispersion. The alkali cation exchange [schematically represented in Fig. 1(a)] was achieved by dispersing $4 \times 10^{-4} \mathrm{~mol}$ of the metal hydroxide per milligram of o-MWNT in $2 \mathrm{ml}$ high performance liquid chromatography (HPLC) grade de-ionized water. Solutions were thoroughly mixed in an ultrasonic bath and then washed with pure water until the filtrate was $p \mathrm{H}$ neutral, ensuring the removal of excess $\mathrm{LiOH}$. Herein, lithium treated o-MWNT will be referred to as $\mathrm{Li}^{+} \mathrm{MWNT}$.

The as-purchased carbon fiber fabric (Ballard, US) was extremely hydrophobic and initially little or no wetting between it and the o-MWNT solution occurred. To render the carbon fiber hydrophilic, it was subject to a microwave oxygen plasma treatment. The oxygen plasma treatment partially oxidizes the carbon fiber surface, creating oxygen containing moieties, which enable hydrogen bonding and therefore wetting between the o-MWNT solution and the carbon felt surface. Additionally, hydrogen bonding will result in strong adhesion between the carbon fiber and the o-MWNT layer. The plasma treated carbon felt was dipped in o-MWNT and $\mathrm{Li}^{+} \mathrm{MWNT}$ solutions, removed and baked at $100{ }^{\circ} \mathrm{C}$ for $20 \mathrm{~min}$ to remove residual water. 

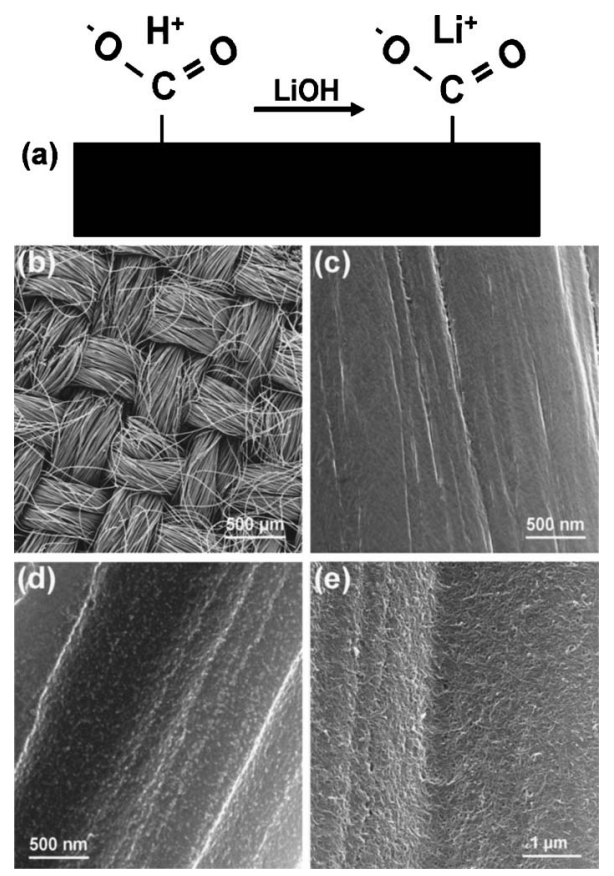

FIG. 1. (a) Schematic of the cation exchange process. Scanning electron microscopy images of (b) macroscopic morphology of carbon fiber fabric, (c) high magnification view of pristine carbon fiber, (d) plasma-oxidized carbon fiber, and (e) carbon fiber after the o-MWNT dip coating process.

Figures 1(b)-1(e) show scanning electron microscopy images of the carbon fiber fabric substrates before and after the coating process. Figure 1(b) shows the macroscopic morphology of the carbon fiber fabric made up of individual carbon fibers $7.5 \mu \mathrm{m}$ in diameter. Figure 1(c) shows the carbon fiber at a higher magnification. This is compared to the same fiber after oxygen plasma treatment [Fig. 1(d)] which shows evidence of surface damage and inhomogeneity at the nanoscale. After o-MWNT coating, individual o-MWNT can clearly be seen in Fig. 1(e) as a uniform layer across the whole carbon fiber surface. Carbon fiber fabric coated in $\mathrm{Li}^{+} \mathrm{MWNT}$ appeared identical to the o-MWNT coated tubes (not shown).

Pristine, plasma treated o-MWNT coated and $\mathrm{Li}^{+} \mathrm{MWNT}$ coated carbon fiber fabrics were characterized for their FE properties at $10^{-6} \mathrm{mbar}$ in a simple diode configuration. A $5 \mathrm{~mm}$ diameter spherical stainless steel anode was placed typically $4 \mathrm{~mm}$ above the substrate. The anode voltage (dc) was incremented in $30 \mathrm{~V}$ steps and current readings were taken as an average of 100 measurements obtained once the voltage had stabilized. The macroscopic electric field $(E)$ was calculated by dividing the applied voltage by the electrode gap. The threshold field $\left(E_{\mathrm{th}}\right)$ was measured as the macroscopic electric field at which an emission current of $1 \mathrm{nA}$ was detected. The average $E_{\mathrm{th}}$ was calculated over six different emission sites on each sample, with the voltage cycled up and down five times at each site. Similar measurements have also been performed on carbon felts onto which CNTs were directly grown using plasma based chemical vapor deposition. ${ }^{19}$

The FE results are summarized in Fig. 2. Figure 2(a) shows the FE current as a function of the applied macroscopic field for the four differently treated carbon fiber fabric samples. Numerical data are summarized in Table I. The pristine carbon fiber fabric had an $E_{\mathrm{th}}$ of $0.42 \mathrm{~V} / \mu \mathrm{m}$ with a hysteresis between the up and down cycles at $1 \mathrm{nA}$ of Downloaded 30 Mar 2009 to 131.227 .178 .132 . Redistribution subject

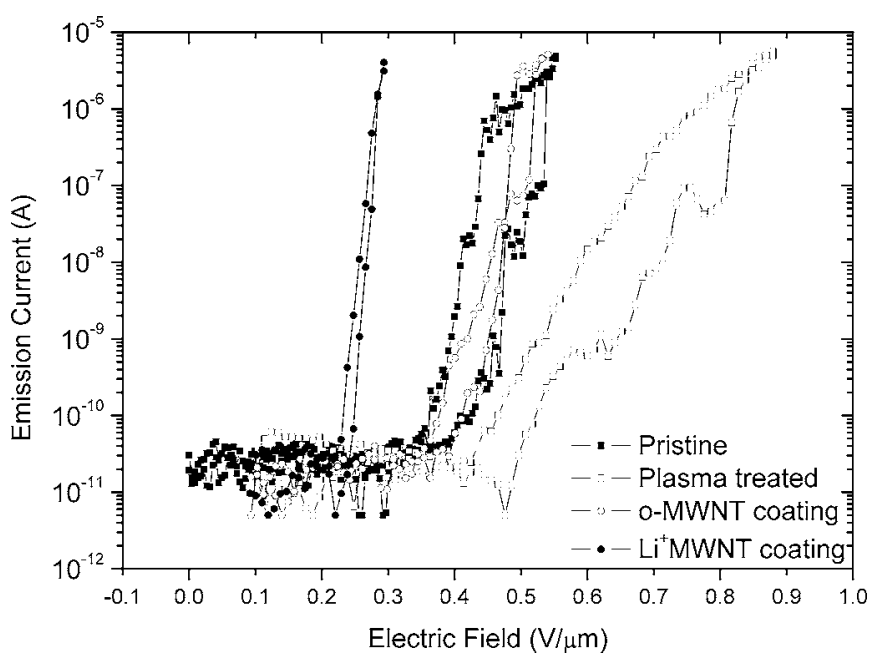

FIG. 2. Comparison of field emission current-field curves for pristine, plasma treated, o-MWNT coated and $\mathrm{Li}^{+} \mathrm{MWNT}$ coated carbon fiber fabrics.

$0.20 \mathrm{~V} / \mu \mathrm{m}$, and a statistical error of $\pm 0.03 \mathrm{~V} / \mu \mathrm{m}$ after averaging. After oxygen plasma treatment, $E_{\mathrm{th}}$ is observed to rise to $0.58 \mathrm{~V} / \mu \mathrm{m}$ with a larger hysteresis of $0.23 \mathrm{~V} / \mu \mathrm{m}$ and larger statistical error of $\pm 0.15 \mathrm{~V} / \mu \mathrm{m}$. This increase in $E_{\text {th }}$ can be attributed to the increase in the work function of the carbon fiber upon plasma treatment. Ago et al. ${ }^{10}$ have previously shown that the work function of pristine MWNT $(\sim 4.4 \mathrm{eV})$ is close to that of graphite $(\sim 4.4 \mathrm{eV})$ and oxygen plasma treatment increases the work function to $4.8 \mathrm{eV}$. Since carbon fibers and MWNT both comprise graphitized carbon, a similar increase in work function of carbon fibers could be expected. Owing to the porous nature of the carbon fiber fabric, it was not possible to make a direct measurement of the work function using ultraviolet photoelectron spectroscopy. The slight increase in hysteresis and substantial increase in error across the sample are indicative of the uneven etching of the carbon fiber by the plasma oxidization process [Fig. 1(c)].

Coating the plasma treated carbon fiber with o-MWNT results in a decrease in $E_{\text {th }}$ back down to $0.42 \mathrm{~V} / \mu \mathrm{m}$ with a hysteresis of $0.08 \mathrm{~V} / \mu \mathrm{m}$, and a statistical error of $\pm 0.09 \mathrm{~V} / \mu \mathrm{m}$. This significant reduction in hysteresis, error and $E_{\mathrm{th}}$, is indirect evidence that $\mathrm{FE}$ occurs predominantly from the o-MWNT themselves. The reduction in $E_{\text {th }}$ compared with the plasma treated fiber is attributed to the modification of the surface geometry of the fibers by the o-MWNT, resulting in an overall increase in geometric enhancement and therefore a higher local field at the emitting sites. However, since the work function of o-MWNT $(5.0 \mathrm{eV})($ Ref. 18) is much larger than that of pristine graphitic carbon fiber $(4.4 \mathrm{eV})$, the beneficial effect of geomet-

TABLE I. Summary of numerical results.

\begin{tabular}{lccccc}
\hline \hline & $\begin{array}{c}E_{\text {th }} \\
(\mathrm{V} / \mu \mathrm{m})\end{array}$ & $\begin{array}{c}\text { Error } \\
(\mathrm{V} / \mu \mathrm{m})\end{array}$ & $\begin{array}{c}\text { Hysteresis } \\
(\mathrm{V} / \mu \mathrm{m})\end{array}$ & $\begin{array}{c}\text { Work } \\
\text { function } \\
(\mathrm{eV})\end{array}$ & Ref. \\
\hline Pristine carbon fiber & 0.42 & \pm 0.03 & 0.20 & 4.4 & 10 \\
Plasma treated fiber & 0.58 & \pm 0.15 & 0.23 & 4.8 & 10 \\
o-MWNT coated fiber & 0.42 & \pm 0.09 & 0.08 & 5.1 & 18 \\
Li $^{+}$MWNT coated fiber & 0.25 & \pm 0.02 & 0.04 & 4.5 & 18 \\
\hline \hline
\end{tabular}


ric field enhancement is offset by the increase in work function.

Coating plasma treated carbon fiber fabric with $\mathrm{Li}^{+} \mathrm{MWNT}$ results in a further decrease of $E_{\text {th }}$ down to $0.25 \mathrm{~V} / \mu \mathrm{m}$ with a hysteresis of $0.04 \mathrm{~V} / \mu \mathrm{m}$ and statistical error of $\pm 0.02 \mathrm{~V} / \mu \mathrm{m}$ across the sample substrate. This improvement is attributed to the change in work function from 5.0 to $4.6 \mathrm{eV}$ between the o-MWNT and the $\mathrm{Li}^{+} \mathrm{MWNT}$, respectively. ${ }^{18}$ The statistical error is an indicator of the uniformity of emission across the substrate. In this case, the extremely low error suggests that there is a very little variation in the emission properties across the entire $>1 \mathrm{~cm}^{2}$ substrate. It should also be noted that the hysteresis for the $\mathrm{Li}^{+} \mathrm{MWNT}$ coated samples is also exceptionally low, indicative of the stability of the emitter during FE.

In summary, carbon fiber fabric was surface oxidized and dip coated in o-MWNT and $\mathrm{Li}^{+} \mathrm{MWNT}$ and the field emission properties were investigated. The threshold field was observed to vary depending on the process applied to the carbon fiber fabric, ultimately leading to a substantial reduction in threshold field from $0.42 \mathrm{~V} / \mu \mathrm{m}$ for o-MWNT to $0.25 \mathrm{~V} / \mu \mathrm{m}$ for $\mathrm{Li}^{+} \mathrm{MWNT}$. This marked reduction is attributed to the reduction in work function from 5.0 to $4.6 \mathrm{eV}$. An associated decrease in hysteresis and an increase in emission uniformity across the sample were also observed (Table I). This method provides a simple, cheap, and scalable way of creating high performance, large area, and flexible field emitting electrodes.

The authors would like to thank EPSRC (UK) for funding this research via the CBE and Portfolio Partnership programmes.
${ }^{1}$ W. A. de heer, A. Chatelain, and D. Ugarte, Science 270, 1179 (1995).

${ }^{2}$ A. G. Rinzler, J. H. Hafner, P. Nikolaev, L. Lou, S. G. Kim, D. Tomanek, P. Nordlander, D. T. Colbert, and R. E. Smalley, Science 269, 1550 (1995).

${ }^{3}$ W. B. Choi, D. S. Chung, J. H. Kang, H. Y. Kim, Y. W. Jin, I. T. Han, Y. H. Lee, J. E. Jung, N. S. Lee, G. S. Park, and J. M. Kim, Appl. Phys. Lett. 75, 3129 (1999).

${ }^{4}$ R. C. Smith, J. D. Carey, C. H. P. Poa, D. C. Cox, and S. R. P. Silva, J. Appl. Phys. 95, 3153 (2004).

${ }^{5}$ R. H. Baughman, A. A. Zakhidov, and W. A. de heer, Science 297, 787 (2002).

${ }^{6}$ J. M. Bonard, T. Stockli, F. Maier, W. A. de Heer, A. Chatelain, J. P. Salvetat, and L. Forro, Phys. Rev. Lett. 81, 1441 (1998).

${ }^{7}$ Q. H. Wang, A. A. Setlur, J. M. Lauerhaas, J. Y. Dai, E. W. Seelig, and R. P. H. Chang, Appl. Phys. Lett. 72, 2912 (1998).

${ }^{8}$ R. C. Smith, D. C. Cox, and S. R. P. Silva, Appl. Phys. Lett. 87, 103112 (2005).

${ }^{9}$ X. Guo and S. R. P. Silva, Electron. Lett. 40, 1113 (2004).

${ }^{10}$ H. Ago, T. Kugler, F. Cacialli, W. R. Salaneck, M. S. P. Shaffer, A. H. Windle, and R. H. Friend, J. Phys. Chem. B 103, 8116 (1999).

${ }^{11}$ R. H. Fowler and L. Nordheim, Proc. R. Soc. London, Ser. A 119, 173 (1928).

${ }^{12}$ A. Wadhawan, R. E. Stallcup, and J. M. Perez, Appl. Phys. Lett. 78, 108 (2001).

${ }^{13}$ D. H. Kim, H. R. Lee, M. W. Lee, J. H. Lee, Y. H. Song, J. G. Jee, and S. Y. Lee, Chem. Phys. Lett. 355, 53 (2002).

${ }^{14}$ N. Park, S. Han, and J. Ihm, J. Nanosci. Nanotechnol. 3, 179 (2003).

${ }^{15}$ S. Dimitrijevic, J. C. Withers, V. P. Mammana, O. R. Monteiro, J. W. Ager, I. G. Brown, Appl. Phys. Lett. 75, 2680 (1999).

${ }^{16}$ F. Jin, Y. Liu, and C. M. Day, Appl. Phys. Lett. 88, 163116 (2006).

${ }^{17}$ W. Feng, K. Kamide, and F. Zhou, Jpn. J. Appl. Phys., Part 2 43, L36 (2004).

${ }^{18}$ N. P. Blanchard, R. A. Hatton, and S. R. P. Silva (to be published).

${ }^{19}$ J. M. Rosolen, S. Tronto, M. S. Marchesin, E. C. Almeida, N. G. Ferreira, C. H. P. Poa, and S. R. P. Silva, Appl. Phys. Lett. 88, 083116 (2006). 